\title{
The Newsboy Model: Changes in Risk and Price
}

JORGE IBARRA-SALAZAR

jaibarra@itesm.mx

Department of Economics, Instituto Tecnológico y de Estudios Superiores de Monterrey, Sucursal de Correos J, Monterrey NL, 64849, Mexico

Received February 14, 2003; Revised June 28, 2004

\begin{abstract}
In this paper I extend the literature related with the newsboy model by analyzing the effect on orders of changes in risk and price. I show that risk aversion is necessary and sufficient condition for the newsboy to decrease orders when the demand suffers an FSD deterioration in risk, and analyze changes in price including an effect, which has been ignored in the literature.
\end{abstract}

Key words: demand uncertainty, newsboy model, changes in risk

JEL Classification No.: D8

\section{Introduction}

The model considered in this paper consists of a firm that faces a given price and must select output ex-ante, that is, before the random demand is observed. If actual demand is less than output, this lesser amount constitutes sales, but if demand exceeds output, only the quantity produced can be sold. Because output and demand may in general not be equal, Leland [1972] considers this a disequilibrium model. In the related literature, this model has been motivated in different manners: as representing a firm that selects output or capacity when facing price regulation, or a newsboy whose problem is to decide on the amount of orders, given an exogenous price. The classical newsboy inventory problem consists in determining the product order quantity that maximizes expected profit in a single period with risky demand. The problem considers a single perishable item and assumes that in case of a shortage, unsatisfied demand is lost.

The decision variable in the model could be interpreted as the number of units to order from a supplier, as in the newsboy's story, as the capacity decision of an entrepreneur, or as the output decision of a firm. The exogenous price could be understood as the price taken by firms that act as competitive firms in imperfect markets, so as to avoid the uncertainties of price competition [Hymans, 1966; Baron, 1971], as the ceiling price fixed by a regulatory agency [Persky, 1990], or as the fixed price, as in the newsboy model applied in both economics and operational research.

The effect of changes in risk for the newsboy model has been studied in the operational research literature, and characterized by parametric changes, such as the standard deviation or mean of the demand distribution [Lau and Lau, 1997]. Simple spreads of the random 
demand, applying the transformation approach, have been studied as well [Gerchak and Mossman, 1992].

The economics literature has also treated the effect on orders of changes in risk. Kanbur [1982] analyzed increases in risk but only for the special case of the quadratic utility function. Eeckhoudt et al. [1995] provide sufficient conditions to determine the effect on optimal order of mean preserving increases in risk and simple spreads across the optimal order. Dionne and Mounsif [1996] extend Eeckhoudt et al. [1995] by analyzing different types of mean preserving spreads (MPS) on the distribution of the demand. Comprehensive literature reviews of the newsboy model are presented in Khouja [1999] and Silver et al. [1998].

In a recent contribution related with comparative statics under uncertainty, Athey [2002] ${ }^{1}$ characterized log-supermodularity and single crossing properties in stochastic problems. In particular, whenever the objective function is given by $U(x, \theta)=\int u(x, s) f(s, \theta) d s$, where $x$ is a choice vector, $s$ is a random variable and $\theta$ an exogenous parameter, Athey [2002] analyzed conditions on the payoff function and the probability density, the primitives, under which the agent's optimal choice of $x$ is nondecreasing in $\theta$.

This paper contributes to the existing literature by studying the effect of a first-degree stochastic dominance (FSD) shift in the distribution of the demand. The main contribution consists in showing that risk aversion is both necessary and sufficient condition for the firm to decrease optimal output whenever the demand undergoes an FSD increase in risk. This result is then applied to analyze the effect of price changes on the decision variable. While this last issue has been studied [Hymans, 1966; Eeckhoudt et al., 1995], the effect that price might exert on the demand distribution has been overlooked. For the case of risk aversion, Hymans [1966] demonstrates that downward-sloping supply is possible, whereas Eeckhoudt et al. [1995] show that decreasing partial relative risk aversion is sufficient for a positive relationship between price and orders. This latter result, however, assumes implicitly that price does not have any influence on the demand distribution. I extend their result by explicitly recognizing the effect that a change in price might have on the distribution of the random demand: Price-unresponsive demand is a special case in my model.

The remainder of the paper is organized as follows. Section 2 describes the model and the notation used; Section 3 presents the result concerning the effects of changes in risk; Section 4 analyzes the effects on optimal output of changes in the exogenous price; and Section 5 concludes.

\section{The model}

This paper considers a firm, or newsboy, who in a single period faces a given price $(p)$ and chooses output $(q)$ so as to maximize the expected utility of profits, $E[u(\pi(x, q ; p))]$, where $E$ denotes the expectation operator. The function $u(\pi(x, q ; p))$ represents a von NeumannMorgenstern utility function, assumed to be twice differentiable with $\mathrm{u}^{\prime}(\pi)>0$ for all $\pi$. $\pi(q, x ; p)=p \min \{x, q\}-c(q)-\varphi$ denotes profits, which equivalently could be written as:

$$
\pi(q, x ; p)= \begin{cases}\pi_{-}=p x-c(q)-\varphi & \text { if } x \leq q \\ \pi_{+}=p q-c(q)-\varphi & \text { otherwise }\end{cases}
$$


$x$ is the random demand with support $[\underline{x}, \bar{x}], c(q)$ represents the variable cost function, twice differentiable with $c(0)=0, c^{\prime}(q)>0$ for all non-negative $q$, and known with certainty by the producer, and $\varphi$ the fixed cost. The price is determined exogenously, below the price that the firm would choose without any constraint. The distribution function of the random demand, $x$, is denoted as $F(x ; p)$, assumed differentiable with respect to both $x$ and $p$, such that $\frac{\partial F(x ; p)}{\partial p} \geq 0$, whenever $0<F(x ; p)<1$, and with the strict inequality over a set of $x$ of positive probability measure. That is, the exogenous price is a parameter in the distribution of the demand, such that it causes an FSD deterioration in risk when it is increased. ${ }^{2}$ It is further assumed that production in excess of demand will perish, and if excess demand exists, it remains unfulfilled.

Note that price enters both the payoff and the distribution functions. Putting this model in Athey's [2002] framework, the objective function is given by $\mathrm{U}(q, p)=\int u(x, q, p) f(x, p)$ $\mathrm{d} x$. Some properties of such model, related with single crossing of the primitives, are studied in Athey [1996].

The model in this paper is more general than the classical newsboy model since it considers the expected utility of profits, instead of expected profits, and an increasing cost function, instead of constant unit cost. Furthermore, the model is similar to that of Dionne and Mounsif [1996] and differs slightly to that of Eeckhoudt et al. [1995] since they assume linear costs, include the possibility of obtaining additional quantities of the product when demand exceeds the original order, at a higher cost, and allow for a positive salvage value of excess inventory. These differences are not crucial in my results.

The firm wishes to maximize

$$
E[u(\pi(x, q ; p))]=\int_{\underline{\mathbf{x}}}^{q} u\left(\pi_{-}\right) d F(x ; p)+u\left(\pi_{+}\right) \int_{q}^{\bar{x}} d F(x ; p) \equiv \bar{u}(q) .
$$

Assuming an interior solution exists, the first-order condition (FOC) is given by:

$$
\bar{u}_{q}(q)=-c^{\prime}(q) \int_{\underline{\mathrm{x}}}^{q} u^{\prime}\left(\pi_{-}\right) d F(x ; p)+\left(p-c^{\prime}(q)\right) u^{\prime}\left(\pi_{+}\right) \int_{q}^{\bar{x}} d F(x ; p)=0 .
$$

From (2) it follows that if the firm produces an optimal positive output, then the price should be set equal or above marginal cost. Concavity of the utility function (risk aversion) and convexity of the cost function are sufficient for the second order condition (SOC) to be satisfied, $\bar{u}_{q q}(q)<0$. Under those conditions, $p \min \{x, q\}-c(q)-\varphi$ is a concave function of $q$ for any $x$, and then $u(p \min \{x, q\}-c(q)-\varphi)$ is also concave. Since the sum of concave functions is concave, therefore $\bar{u}(q)$ is concave.

The firm shuts down if the expected utility of profits, with output equal to zero, is larger than the expected utility corresponding to any positive output. That is, when $u(-\varphi)>\bar{u}(q)$ for $q>0$. The concavity of the expected utility of profits function implies that utility of expected profits is greater than expected utility of profits, or in symbols: $u(E[\pi(x, q ; p)])>$ $\bar{u}(q)$. Thus, a sufficient condition for $q=0$ is $u(-\varphi)>u(E[\pi(x, q ; p)])$. Since the utility function is strictly increasing, this latter condition is equivalent to $-\varphi>E[\pi(x, q ; p)]$ $=p E[\min \{x, q\}]-c(q)-\varphi$, or that $p E[\min \{x, q\}]<c(q)$. Hence, a sufficient condition for $q=0$ is that the expected sales revenue be smaller than the variable cost. Furthermore, a necessary condition for $q>0$ is that expected sales revenue be greater than variable cost. 


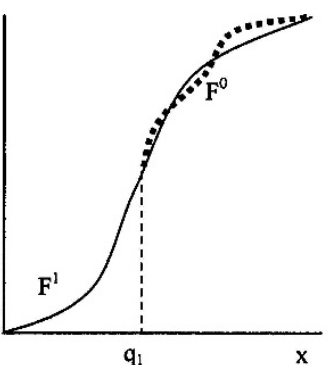

a. Marginal increase in risk restricted to $\left[\mathrm{q}_{1}, \overline{\mathrm{x}}\right]$

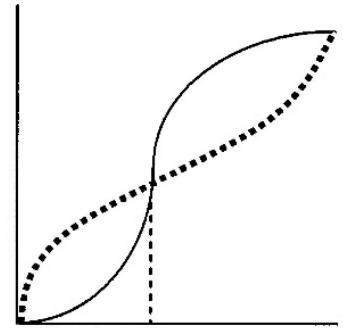

c. Simple spread across $\mathrm{q}_{1}$

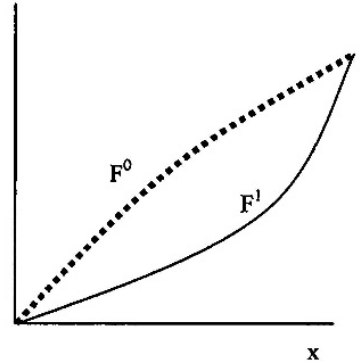

e. First degree stochastic dominance

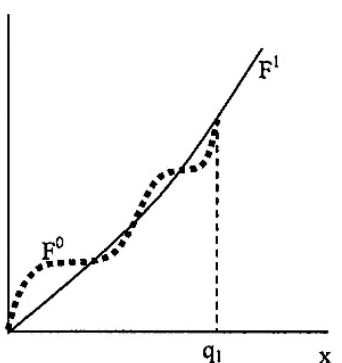

b. Marginal increase in risk restricted to $\left[x, q_{1}\right]$

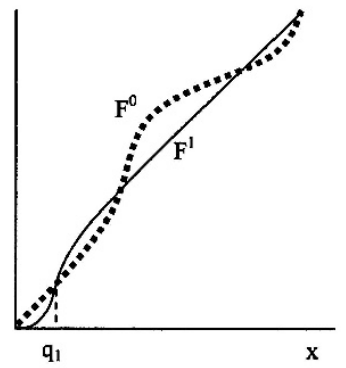

d. Mean preserving spread with $F^{0} \geq F^{1}$ for all $x \leq q_{1}$

Figure 1. Various types of changes in risk.

\section{Marginal changes in risk}

The marginal effect of uncertainty is represented as the change in the optimal output brought about by a shift in the probability distribution of the random demand. Eeckhoudt et al. [1995], and Dionne and Mounsif [1996] have analyzed the effects of restricted mean preserving increases in risk and simple spreads across the optimal output for the newsboy model. Figure 1, exhibits a, b, c and d, presents the different types of shifts studied in those papers. It has been shown that prudence and risk aversion are sufficient conditions in order to determine the effect of a restricted mean preserving increase in risk and of a simple spread across the optimal output, respectively. Proposition 1 below, presents a result that contributes 
to previous studies in two ways: First, by considering a type of change in risk not yet studied in the newsboy literature, an FSD shift in the distribution of the demand (figure 1, exhibit e); and second, by showing that risk aversion is not only sufficient, but also a necessary condition for the firm to decrease output when the demand experiences an FSD increase in risk. Because of such result, the proposition defines the risk averse newsboy under demand uncertainty: He decreases output whenever faced with an FSD deterioration in risk. Before presenting Proposition 1, I define an FSD risk change.

Definition (Hadar and Russell [1971]): The distribution $f^{1}$ is said to be larger than $f^{0}$ in the sense of FSD, if $F^{1}(x) \leq F^{0}(x)$ for all $x \in[\underline{x}, \bar{x}]$ and the strict inequality holds for some $x$.

Following Hadar and Russell [1978], an FSD shift could be motivated by a change in an exogenous parameter, for example, consumers' income or advertising expenditures by the firm. The case of changes in the exogenous price is analyzed in the next section. The approach followed in Proposition 1 is very similar to that of Hadar and Seo [1992] for the case of the competitive firm under price uncertainty, and related with the general approach to comparative statics under uncertainty in Athey [2002]. Following Athey [2002], we impose conditions on primitives, the probability distribution and the payoff function, in order to determine the effect in the decision variable of a shift in the distribution. In particular, we impose an FSD increase in risk in the distribution of demand, which is weaker than logsupermodularity of the cumulative distribution, and that the second derivative of the utility function is negative for all $x$. Given the condition on the cumulative distribution, I show that the condition on the utility function is both necessary and sufficient for the optimal output to decrease.

Let $q_{1}$ and $q_{0}$ be the optimal outputs under $F^{1}(x ; p)$ and $F^{0}(x ; p)$ respectively.

Proposition 1: Given any $F^{1} F S D F^{0}$, any convex $c(q)$, and any admissible constrained price $e^{3}$ under both $F^{1}(x ; p)$ and $F^{0}(x ; p)$, then $q_{1}>q_{0}$ if and only if $u^{\prime \prime}(\pi(x, q ; p))<0$ for all $x$.

Proof. Sufficiency. From (2) the FOC under $F^{1}(x ; p)$ can be written as:

$$
\bar{u}_{q}^{1}(q)=-c^{\prime}(q) \int_{\underline{x}}^{q} u^{\prime}\left(\pi_{-}\right) d F^{1}(x ; p)+\left(p-c^{\prime}(q)\right) u^{\prime}\left(\pi_{+}\right) \int_{q}^{\bar{x}} d F^{1}(x ; p)=0 .
$$

Evaluating (4) at $q=q_{0}$, and subtracting the FOC under $F^{0}(x ; p)$, it is obtained:

$$
\begin{aligned}
\bar{u}_{q}^{1}\left(q_{0}\right)= & c^{\prime}\left(q_{0}\right) \int_{\underline{x}}^{q_{0}} u^{\prime}\left(\pi_{-}\right) d\left(F^{0}(x ; p)-F^{1}(x ; p)\right) \\
& -u^{\prime}\left(\pi_{+}\right)\left(p-c^{\prime}\left(q_{0}\right)\right) \int_{q_{0}}^{\bar{x}} d\left(F^{0}(x ; p)-F^{1}(x ; p)\right) .
\end{aligned}
$$


Integrating the above expression by parts and simplifying yields:

$$
\begin{aligned}
\bar{u}_{q}^{1}\left(q_{0}\right)= & -p c^{\prime}\left(q_{0}\right) \int_{\underline{x}}^{q_{0}} u^{\prime \prime}\left(\pi_{-}\right)\left(F^{0}(x ; p)-F^{1}(x ; p)\right) d x \\
& +p u^{\prime}\left(\pi_{+}\right)\left(F^{0}\left(q_{0} ; p\right)-F^{1}\left(q_{0} ; p\right)\right) .
\end{aligned}
$$

Given that $F_{1} \operatorname{FSD} F^{0}$, and the assumption that $u(\pi(x, q ; p))$ is increasing and concave, expression (5) is positive, so that $\bar{u}_{q}^{1}\left(q_{0}\right)>0$. Hence, output under $F^{1}(x ; p)$ is greater than output under $F^{0}(x ; p)$.

Necessity. It has to be proved that for any $F^{1}$ FSD $F^{0}$, any convex $c(q)$, and any admissible price $p$, under both $F^{1}(x ; p)$ and $F^{0}(x ; p)$, if $q_{1}>q_{0}$ then $u^{\prime \prime}(\pi(x, q ; p))<0$ for all $x$. See Appendix

\section{Changes in price}

In this section, the effect of a change in the exogenous price, in such a way that it remains admissible, is analyzed. Since price enters not only the payoff but also the distribution function, changing it will alter both. The effect of an increase in price, therefore, consists of a payoff effect $(\eta)$ and a distribution effect $(\psi)$. A price increase raises producer revenues regardless of the quantity sold. The additional revenue is proportional to $p x$ if sales equal the quantity demanded, and proportional to $p q$ when quantity produced falls short of demand.

In order to determine this effect on optimal output, the sign of $\frac{\partial q}{\partial p}=-\frac{\bar{u}_{q p}(q)}{\bar{u}_{q q}(q)}$ needs to be established. Since, by the SOC, the denominator is negative, the sign indicating the qualitative effect of a price change is the same as that of $\bar{u}_{q p}(q)$. Taking the derivative with respect to $p$, from (2), this can be written as:

$$
\begin{aligned}
\bar{u}_{q p}(q)= & \eta+\Psi, \text { where } \\
\eta \equiv & -c^{\prime}(q) \int_{\underline{x}}^{q} u^{\prime \prime}\left(\pi_{-}\right) x d F(x ; p)+\left(p-c^{\prime}(q)\right) u^{\prime \prime}\left(\pi_{+}\right) q \int_{q}^{\bar{x}} d F(x ; p)+u^{\prime}\left(\pi_{+}\right) \\
& \times \int_{q}^{\bar{x}} d F(x ; p), \quad \text { and } \\
\Psi= & p c^{\prime}(q) \int_{\underline{x}}^{q} u^{\prime \prime}\left(\pi_{-}\right) \frac{\partial F(x ; p)}{\partial p} d x-p u^{\prime}\left(\pi_{+}\right) \frac{\partial F(q ; p)}{\partial p} .
\end{aligned}
$$

Note that the term $\eta$ includes the effect of the change in price via the payoff function, whereas $\Psi$ presents the effect brought about by the distribution function. In the framework of Athey [2002], those are the effects on the decision variable looking at the primitive functions: The payoff and the distribution of the random variable. In general, the sign of $\bar{u}_{q p}(q)$ is indeterminate since it depends on both the responsiveness of the demand distribution to changes in price, $\frac{\partial F(x ; p)}{\partial p}$, and on the properties of the partial measure of risk aversion, $r_{p}(\pi)=\left[r_{A}(\pi)\right] p x$, where $r_{A}(\pi)$ is the Arrow-Pratt absolute index of risk aversion. In particular, if $r_{P}(\pi)$ is assumed to be non-increasing in $x,{ }^{4}$ substituting for this risk aversion 
measure in $\eta$, the following inequality is obtained:

$$
\begin{aligned}
\eta \geq & r_{p}(\pi)\left[c^{\prime}(q) \int_{\underline{x}}^{q} u^{\prime}\left(\pi_{-}\right) d F(x ; p)-\left(p-c^{\prime}(q)\right) u^{\prime}\left(\pi_{+}\right) \int_{q}^{\bar{x}} d F(x ; p)\right] \\
& +u^{\prime}\left(\pi_{+}\right) \times \int_{q}^{\bar{x}} d F(x ; p) .
\end{aligned}
$$

Since the term in brackets is equal to zero by virtue of (2), then $\eta>0$. In this paper it is assumed that a marginal increase in price causes an increase in risk, represented by an unfavorable shift in the distribution of demand, that is: $\frac{\partial F(x ; p)}{\partial p} \geq 0$. This assumption is sufficient for $\psi<0$. Provided that $\eta>0$ and $\psi<0$, the total price-effect depends on the relative magnitudes of the payoff effect vs. the distribution effect.

Just as in the certainty case, with linear demand and price regulation, in which the slope of the supply function depends on the price elasticity of the demand [Hadar, 1971], the slope of the supply function under uncertainty depends not only on the partial risk aversion index, but also on the responsiveness of the demand to price changes. If, for example, multiplicative demand uncertainty is assumed $\left(x=h(p) \mu, h^{\prime}(p)<0, E \mu=1\right)$, together with non-increasing $r_{p}(\pi)$, the slope of the supply function could be expected to be positive if the exogenous price is low enough (inelastic region of the demand), and negative for relatively high prices (elastic region).

This result could also be explained by identifying two effects in $\partial q / \partial p$. Writing this as:

$$
\frac{\partial q}{\partial p}=-\frac{\eta}{\bar{u}_{q q}(q)}-\frac{\psi}{\bar{u}_{q q}(q)}
$$

the payoff effect $\left(-\eta / \bar{u}_{q q}(q)\right)$ is the variation in output caused by the price change via the profit function, holding the distribution of demand constant; the distribution effect $\left(-\psi / \bar{u}_{q q}(q)\right)$ represents the change in output motivated only by the change in the distribution of the demand, as caused by the change in price. Eeckhoudt et al. [1995] considered only the former. In general, however, the sign of the supply curve's slope depends on the relative magnitude of these two effects. For example, if price increases, the distribution of the demand will experience an FSD deterioration in risk, causing a decrease in output as indicated by the distribution effect. Provided $r_{p}(\pi)$ is non-increasing, the payoff effect will cause an increase in output in response to the increase in price. Thus, the slope of supply will be positive (negative) if the payoff effect exceeds (is less than) the distribution effect.

This finding supports the result by Hymans [1966] in that the slope of the supply curve for the price-taker firm needs not be positive. In the model developed in this paper such result could be obtained if $r_{p}(\pi)$ is increasing in $x$, or if non-increasing, when the distribution effect is greater than the payoff effect. We summarize the effect of changes in the exogenous price in Proposition 2.

Proposition 2: The payoff effect is positive $(\eta>0)$ if the partial measure of risk aversion is non-increasing. 
The supply function is positively sloped if the payoff effect is greater than the distribution effect, and if the partial measure of risk aversion is non-increasing.

In particular, the result in Eeckhoudt et al. [1995] is obtained if the partial measure of risk aversion is non-increasing and there is no distribution effect, that is, if the distribution of demand is not affected by the change in price.

\section{Conclusions}

The literature related with changes in risk for the newsboy model has presented sufficient conditions to determine the effect on optimal output of mean-preserving marginal changes in risk. I have extended those results by showing that risk aversion is a necessary and sufficient condition for the firm to decrease output when the distribution of the demand undergoes an FSD increase in risk. Because of such relationship of equivalence, this result can be used to characterize the risk-averse newsboy. The type of shifts addressed in Eeckhoudt et al. [1995] and Dionne and Mounsif [1996] could be further analyzed, along the lines of Athey [2002], in order to explore the idea that prudence and risk aversion might be not only sufficient, but also necessary conditions for the firm to decrease output when facing an MPS shift in risk.

An additional issue was the effect of changes in the exogenous price on optimal output. Extending the study by Eeckhoudt et al. [1995], two effects were identified: The payoff effect, defined as the change in output motivated by a variation in the price via the profit function; and the distribution effect, defined as the change in output brought about only by the effect of the price change over the distribution of the demand. Non-increasing partial risk aversion is sufficient for the payoff effect to be positive (output moves in the same direction as price). When isolating this effect from the distribution effect, as in Eeckhoudt et al. [1995], the slope of the supply curve is positive. However, when both payoff and distribution effects are taken together, we have an inconclusive result, since such effects could have opposite impacts on the optimal output.

\section{Appendix: Necessity part of Proposition 1}

Necessity is proved by contradiction. Assume that $\mathrm{u}^{\prime \prime}(\pi(x, q ; p)) \geq 0$ in some nondegenerate interval $I^{\pi}=\left(\pi_{1}, \pi_{2}\right)$ where $\pi_{1}<\pi_{2}$. It will be shown that there exist some convex cost function, $c_{0}(q)$ with $c_{0}^{\prime}(q)>0$, some distributions $F^{1}\left(x ; p_{0}\right)$ and $F^{0}\left(x ; p_{0}\right)$, some optimal output $q_{0}$, and some admissible price $p_{0}$, which is above marginal cost at $q_{0}$, such that $F^{1}$ FSD $F^{0}$ and yet $q_{1} \leq q_{0}$.

Choose any $q_{0}>0$, any positive real $w_{0}$, such that $w_{0}>-\pi_{1}$, and any positive real $p_{0}$, such that $\frac{\pi_{1}+c_{0}\left(q_{0}\right)}{q_{0}}<p_{0}<\frac{\pi_{2}+c_{0}\left(q_{0}\right)}{q_{0}}$. Define the function $c_{0}(q)=\delta q+w_{0}-\delta q_{0}$, with $0<\delta<\min \left\{\frac{w_{0}}{q_{0}}, \frac{q_{0}+w_{0}}{q_{0}}\right\}$, the numbers $a, b$, and the interval $I^{x}$ as follows: $a=\frac{\pi_{1}+c_{0}\left(q_{0}\right)}{p_{0}}$, $b=\frac{\pi_{2}+c_{0}\left(q_{0}\right)}{p_{0}}, I^{x}=\stackrel{q_{0}}{(a, b)}$ with $a<b$.

Let the random demand take the form: $x=h_{0}(p) \mu$, where $h_{0}(p)$ is the riskless demand function, $h_{0}(p)=\gamma\left[\frac{\pi_{2}+w_{0}}{q_{0}}\right]-p$ where $\gamma \geq 2, \mu$ is a non-negative random variable with distribution $G^{0}(\mu)$, and $E[\mu]=1$. 
Then, it follows that ${ }^{5}$ :

(i). The cost function is defined over the positive real numbers and it is increasing in that domain.

(ii). Price is above marginal cost at $q_{0}\left(p_{0}>c_{0}^{\prime}\left(q_{0}\right)\right)$.

(iii). $q_{0}$ pertains to the set $(a, b)$.

(iv). The utility function is convex $\left(u^{\prime \prime}(\pi(x ; q ; p)) \geq 0\right)$ for all $x \in(\mathrm{a}, \mathrm{b})$, whenever $\pi_{1}<\pi$ $(x, q ; p)<\pi_{2}$ and $\pi(x ; q ; p)=p_{0} x-c_{0}\left(q_{0}\right)$.

(v). The riskless demand is positive when evaluated at $p_{0}$.

(vi). The price $p_{0}$ is admissible.

Now define:

$$
\begin{aligned}
\alpha & \equiv c_{0}^{\prime}\left(q_{0}\right) \int_{\underline{x}}^{q_{0}} u^{\prime}\left(\pi_{-}\right) d x>0, \\
\beta & \equiv u^{\prime}\left(\pi_{+}\right)\left(P_{0}-c_{0}^{\prime}\left(q_{0}\right)\right) \int_{q_{0}}^{b} d x>0, \\
B & \equiv \frac{\beta}{\alpha} q_{0}+\left(b-q_{0}\right)>0 .
\end{aligned}
$$

Consider a random variable with density $f^{0}$ and distribution $F^{0}$, such that:

$$
B f^{0}(x ; p)= \begin{cases}\frac{\beta}{\alpha} & \underline{x} \leq x<q_{0} \\ 1 & q_{0} \leq x \leq b \\ 0 & \text { elsewhere }\end{cases}
$$

Thus, $F^{0}$ is the distribution of the demand, as induced by $\mu$. Note further that $F^{0}$ is strictly increasing and continuous in $[\underline{x}, b]$. Consider the FOC $(2)$ under $F^{0}$, as constructed in (6) above:

$$
\begin{aligned}
\bar{u}_{q}^{0}(q) & =-c_{0}^{\prime}\left(q_{0}\right) \int_{\underline{x}}^{q_{0}} u^{\prime}\left(\pi_{-}\right) d F^{0}\left(x ; p_{0}\right)+u^{\prime}\left(\pi_{+}\right)\left(p_{0}-c_{0}^{\prime}\left(q_{0}\right)\right) \int_{q}^{\bar{x}} d F^{0}\left(x ; p_{0}\right) \\
& =-c_{0}^{\prime}\left(q_{0}\right) \int_{\underline{x}}^{q_{0}} u^{\prime}\left(\pi_{-}\right) B^{-1} \frac{\beta}{\alpha} d x+u^{\prime}\left(\pi_{+}\right)\left(p_{0}-c_{0}^{\prime}\left(q_{0}\right)\right) \int_{q_{0}}^{b} B^{-1} d x . \\
& =B^{-1} \frac{\beta}{\alpha}(-\alpha)+B^{-1} \beta=0 .
\end{aligned}
$$

Then, $q_{0}$ is the optimal output under $F^{0}$.

Given that $q_{0} \in(a, b)$, the distribution $F^{1}$ is now constructed in such a way that $F^{1}$ FSD $F^{0}$, with $\underline{x}<a<a_{1}<b_{1}<q_{0}<b<\bar{x}$. Let $F^{1}\left(x ; p_{0}\right)=F^{0}\left(x ; p_{0}\right)-Z\left(x ; p_{0}\right)$, with $Z\left(x ; p_{0}\right)$ continuous for $x>0, Z\left(x ; p_{0}\right)>0$ for $a_{1}<x<b_{1}$, and $Z\left(x ; p_{0}\right)=0$ for $x \leq a_{1}$ and $x \geq b_{1}$. This construction is possible because $F^{0}$ is increasing and continuous. 
Evaluating the FOC under $F^{1}$ at $q=q_{0}$, and subtracting $\bar{u}_{q}^{0}\left(q_{0}\right)$ yields:

$$
\begin{aligned}
\bar{u}_{q}^{1}\left(q_{0}\right)= & c_{0}^{\prime}\left(q_{0}\right) \int_{\underline{x}}^{q_{0}} u^{\prime}\left(\pi_{-}\right) d\left(F^{0}\left(x ; p_{0}\right)-F^{1}\left(x ; p_{0}\right)\right) \\
& -u\left(\pi_{+}\right)\left(p_{0}-c_{0}^{\prime}\left(q_{0}\right)\right) \int_{q_{0}}^{\bar{x}} d\left(F^{0}\left(x ; p_{0}\right)-F^{1}\left(x ; p_{0}\right)\right) .
\end{aligned}
$$

Given that by construction $F^{1}\left(x ; p_{0}\right)=F^{0}\left(x ; p_{0}\right)$ for $x \leq a_{1}$ and $x \geq b_{1}$, then:

$$
u_{q}^{1}\left(q_{0}\right)=c_{0}^{\prime}\left(q_{0}\right) \int_{a_{1}}^{b_{1}} u^{\prime}\left(\pi_{-}\right) d\left(F^{0}\left(x ; p_{0}\right)-F^{1}\left(x ; p_{0}\right)\right) .
$$

Integrating the last expression by parts:

$$
\bar{u}_{q}^{1}\left(q_{0}\right)=-p_{0} c_{0}^{\prime}\left(q_{0}\right) \int_{a_{1}}^{b_{1}} u^{\prime \prime}\left(\pi_{-}\right)\left(F^{0}\left(x ; p_{0}\right)-F^{1}\left(x ; p_{0}\right)\right) d x \leq 0 .
$$

The inequality follows since $F^{1}$ FSD $F^{0}$ and $u^{\prime \prime}\left(\pi_{-}\right) \geq 0$ in $a_{1} \leq x \leq b_{1}$. Then, with $F^{1}$ the expected utility of profits is maximized at $q=q_{1} \leq q_{0}$.

\section{Acknowledgments}

This paper is a modified version of a chapter from my 1996 Southern Methodist University dissertation. I am grateful for comments, direction, and encouragement received from Josef Hadar and Tae Kun Seo. I also thank Bonnie Palifka and two anonymous referees whose comments substantially improved the paper. The usual disclaimer applies.

\section{Notes}

1. I thank an anonymous referee for making me aware of Susan Athey's paper.

2. In order to motivate this assumption one could consider two specifications of the random demand. Risk could enter the demand in multiplicative form: $x=h(p) \theta$, where $\theta$ is a random variable and $h(p)$ is the riskless demand, with $h^{\prime}(p)<0$. It is customary to assume that the mathematical expectation of $\theta$ equals 1 . In additive form the risky demand would be specified as: $x=h(p)+\mu$, with $\mu$ being a random variable and $h(p)$ being interpreted in the same way as in the multiplicative form. In both cases, the monotonicity assumption on the function $h(p)$ is equivalent to assuming that the induced distribution of $x$ is increasing in price.

3. Admissibility means that the price is below the price that the firm would choose without constraint $\left(p^{m}\right)$. From the second order conditions $p^{m}$ is such that $[x f(x ; p)-p \partial F(x ; p) / \partial p]<0$. Therefore, to ensure admissibility it is sufficient to set the price so as to make $[x f(x ; p)-p \partial F(x ; p) / \partial p] \geq 0$. If that is the case we say that the price is admissible for the given distribution of the demand.

4. The partial risk aversion measure was introduced by Menezes and Hanson [1970]. I am not aware of any piece of evidence indicating that partial risk aversion should be increasing or decreasing. Intuitively, this assumption, also made in Eeckhoudt et al. [1995], means that a given proportional increase in risk, cost constant, will result in a less than proportional increase in the risk premium. Increasing partial risk aversion follows from increasing 
relative risk aversion if final wealth is increasing in its non-random component. In the Newsboy model the non-random component of profits is the cost function. Since an increase in cost reduces profits, in our case increasing relative risk aversion does not imply increasing partial risk aversion. It is interesting to note that for the utility functions: $u(\pi(x))=\ln \pi(x)$ and $u(\pi(x))=[\pi(x)]^{w}, 0<w<1$, both partial and absolute risk aversion are decreasing; whereas for the quadratic utility function, both indexes are increasing.

5. Proof of assertions i. to vi. is available from the author on request.

\section{References}

ATHEY, S. [2002]: “Monotone Comparative Statics under Uncertainty,” Quarterly Journal of Economics, 117(1), $187-223$.

ATHEY, S. [1996]: "Comparative Statics under Uncertainty: Single Crossing Properties and LogSupermodularity," MIT Working Paper No. 96-22.

BARON, D. [1971]: "Demand Uncertainty and Imperfect Competition," International Economic Review, 12, $196-208$.

DIONNE, G. and MOUNSIF, T. [1996]: "Investment under Demand Uncertainty: The Newsboy Problem Revisited," The Geneva Papers on Risk and Insurance Theory, 21, 179-189.

EECKHOUDT, L., GOLLIER, C., and SCHLESINGER, H. [1995]: "Risk-averse (and Prudent) Newsboy," Management Science, 41(5), 786-794.

GERCHAK, Y. and MOSSMAN, D. [1992]: "On the Effect of Demand Randomness on Inventories and Costs," Operational Research, 40, 804-807.

HADAR, J. [1971]: Mathematical Theory of Economic Behavior. Reading: Addison-Wesley.

HADAR, J. and SEO, T.K. [1992]: "General Changes in Uncertainty," Southern Economic Journal, 58, 671-681.

HADAR, J. and RUSSELL, W. [1971]: "Stochastic Dominance and Diversification," Journal of Economic Theory, 3, 288-305.

HADAR, J. and RUSSELL, W. [1978]: “Applications in Economic Theory and Analysis,” in Stochastic Dominance, G.A. Whitmore and M.C. Findlay (Eds.), Lexington, Books, Lexington MA, 295-333.

HYMANS, S. [1966]: “The Price-Taker: Uncertainty, Utility and the Supply Function,” International Economic Review, 7, 346-56.

KANBUR, R. [1982]: “Increases in Risk with Kinked Payoff Functions," Journal of Economic Theory, 27(1), 219-228.

KHOUJA, M. [1999]: “The Single-Period (News-vendor) Problem: Literature Review and Suggestions for Future Research," Omega, The International Journal of Management Science, 27, 537-553.

LAU, H. and LAU, A. [1997]: "Some Results on Implementing a Multi-Item Multi-Constraint Single-Period Inventory Model," International Journal of Production Economics, 48, 121-128.

LELAND, H. [1972]: “Theory of the Firm Facing Uncertain Demand,” American Economic Review, 62, $278-291$. MENEZES, C. and HANSON, D. [1970]: "On the Theory of Risk Aversion,” International Economic Review, 11, 481-487.

PERSKY, A. [1990]: "Stochastic Demand with Uncertain Sales under Various Risk Preferences," Southern Economic Journal, 56(4), 1073-1078.

SILVER, E., PYKE, D., and PETERSON, R. [1998]: Inventory Management and Production Planning and Scheduling, 3rd edn. New York: John Wiley \& Sons. 